\title{
Regulation of porcupine-dependent Wnt signaling is essential for uterine development and function
}

\author{
Omar Farah ${ }^{1,2}$, Steffen Biechele ${ }^{3}$, Janet Rossant ${ }^{4,5}$ \& Daniel Dufort ${ }^{1,2,6}$ \\ ${ }^{1}$ McGill University Health Centre, Montreal, Quebec, Canada, ${ }^{2}$ Division of Experimental Medicine, McGill \\ University, Montreal, Quebec, Canada, ${ }^{3}$ Center for Reproductive Sciences, Department of Obstetrics, Gynecology \\ and Reproductive Sciences, University of California, San Francisco, California, USA, ${ }^{4}$ Department of Molecular \\ Genetics, University of Toronto, Toronto, Ontario, Canada, ${ }^{5}$ Program in Developmental and Stem Cell Biology, \\ Hospital for Sick Children Research Institute, Toronto, Ontario, Canada and ${ }^{6}$ Department of Biology, McGill \\ University, Montreal, Quebec, Canada
}

Correspondence should be addressed to D Dufort; Email: Daniel.dufort@mcgill.ca

\begin{abstract}
Six members of the Wnt family are expressed in the female reproductive tract. Their collective function ensures proper development of the uterus, preparing it for pregnancy during adulthood. Here, we take advantage of the fact that Porcn, a prerequisite for all Wnt secretion, is located on the $\mathrm{X}$ chromosome, to generate females that were mosaic for Porcn throughout the reproductive tract.

Porch $^{\text {flox/+ }}$ females were mated with progesterone receptor $(\mathrm{Pgr})$-Cre males $\left(\mathrm{Pgr}^{\mathrm{Cre} /+}\right)$ to generate females that were heterozygous for Porcupine in all tissues of the female reproductive tract, resulting in mosaicism due to random X-inactivation. We demonstrated that Porcn mosaic females are extremely subfertile and exhibit a large spectrum of phenotypes ranging from morphologically normal uteri to uteri with extremely enlarged cystic glands. Decreased fertility in Porcupine mosaic females was not associated with phenotype severity and was observed regardless of whether or not cystic glands were enlarged. By crossing-in a GFP reporter on the wild-type X chromosome, we were able to correlate endometrial gland hyperplasia with a mostly Porcupine mutant stroma, demonstrating the role of stromal Wnts in the regulation of endometrial gland proliferation. Finally, we demonstrated that fertility issues within mosaic females were due to a reduced response to estrogen and to abnormal Tcf/Lef signaling across the mesometrial-anti-mesometrial axis during the window of implantation.

Reproduction (2018) 155 93-102
\end{abstract}

\section{Introduction}

Human pregnancy is a multi-faceted and highly regulated process that requires the intricate and timely control of numerous signaling pathways. Failure to properly control the pathways involved in pregnancy leads to complications that ultimately result in a change of fertility outcome (Fazleabas et al. 2004, Wang \& Dey 2006, Cha \& Dey 2014, 2015). Recently, Wnt signaling was shown to be essential for various processes, including embryo-uterine crosstalk, implantation, decidualization and placentation (Mohamed et al. 2005, Sonderegger et al. 2010, Dunlap et al. 2011, Franco et al. 2011). Wnts are a group of secreted morphogenic factors, 19 of which are found in both the human and murine genomes. Wht signaling is also involved in the proper development of the female reproductive tract, the completion of which occurs postnatally in murine species (Bartol et al. 1999, Gray et al. 2001). Shortly after birth, the luminal epithelium (LE) within the uterus starts to invaginate toward the endometrial stroma and subsequently forms ductal glands that are referred to as endometrial or uterine glands (Gray et al. 2001, Cooke et al. 2013, Filant \& Spencer 2014).

Porcupine (Porcn) is a gene located on the $\mathrm{X}$ chromosome whose function was shown to be a prerequisite for the release of WNT proteins from the endoplasmic reticulum (ER) into the extra-cellular compartment (van den Heuvel et al. 1993, Kadowaki et al. 1996). Porcupine-dependent palmitoylation on conserved serine residues of all WNT proteins has been shown to be responsible for their transport into the Golgi where they are packaged and sent to the cell membrane for release.

The precise mechanism of porcupine-dependent WNT activity within the female reproductive tract still remains somewhat elusive. Due to the complicated signaling network involving several Wnts expressed in different compartment of the uterus, single Wnt gene mutations paint an incomplete picture of their mode of action. Known redundancies between different Wnts also contribute to this phenomenon. We have previously examined global Wnt ablation, by means of 
a Porcn deletion, in the entire female reproductive tract (Farah et al. 2017). Here, we utilized the underlying genetic makeup of Porcn heterozygous females to generate mosaic females. Since Porcn is located on the $\mathrm{X}$ chromosome, heterozygous females are mosaics due to random $X$-inactivation (Payer 2016). This process, by which one of the female's two $X$ chromosomes is randomly inactivated, results in a mosaic uterus where some cells are mutant for Porcn while others are wildtype. The generation of mosaic individuals results in groups of wildtype cells that are adjacent to mutant cells and allows for the examination of the effect of mutant cells on adjacent wildtype tissues. These mice can also be used to address whether wildtype tissue can rescue adjacent mutant cells.

Here, we report that Porcn mosaic females are extremely subfertile. Histological examination revealed that heterozygosity of Porcn in the uterus results in the formation of large endometrial cysts. This phenotype ranges in severity and seems to correlate to the degree of Porcn mosaicism within the uterine stroma. In addition, these females are extremely subfertile due in part to aberrant Tcf/Lef signaling and a reduced ability to respond to the ovarian hormone estrogen.

\section{Materials and methods}

\section{Animals}

All experimental protocols were approved by the Animal Care Committee of the McGill University Health Centre and were in accordance with regulations established by the Canadian Council on Animal Care. Mice with LoxP sites flanking exon 3 of the Porcn gene (Porcn floxed/floxed) on a mixed background were kindly donated by Dr Janet Rossant. The generation of these mice has been previously described (Biechele et al. 2013). Progesterone receptor-Cre mice $\left(\mathrm{Pgr}^{\mathrm{Cre} /+}\right)$ on a CD1 background were generously provided by $\mathrm{F} J \mathrm{DeMayo}$ and J P Lydon (Baylor College of Medicine) (Soyal et al. 2005). Both strains have previously been reported to demonstrate normal fertility and $\mathrm{Pgr}^{\mathrm{Cre} /+}$ mice have been used in numerous studies to investigate uterine-specific gene function. Homozygous Porch floxedflloxed females were crossed with heterozygous $\mathrm{Pgr}^{\mathrm{Cre} /+}$ males and the offspring were genotyped by tail snip digestion and PCR as previously described (Farah et al. 2017). PorCn ${ }^{\text {floxed/+ }}, \mathrm{PgC}^{\mathrm{Cre} /+}$ females were thus obtained (simplified from here on as Porcn $n^{\Delta /+}$, Porcn ${ }^{\text {mosaics }}$ or Porcn ${ }^{\text {Het }}$ females). For the assessment of the degree of mosaicism, a mouse strain containing a GFP on the X chromosome (Hadjantonakis et al. 1998) was crossed into our mouse strain in order to obtain $X^{\text {Porcn- }}$ floxed/GFP, $P g r^{\text {Cre/+ }}$. For assessment of $\beta$-Catenin activity, a mouse strain containing a Tcf/Lef-LacZ reporter allele (Mohamed et al. 2005) was crossed into the Porcn strain. Vaginal smears were taken from females before tissue collection and processing in order to stage the females into the correct estrous cycle stage as required. The various stages were determined based on the presence and proportion of leukocytes (diestrus), nucleated cells (proestus) and cornified cells (estrus).

\section{Paraffin embedding, sectioning and staining}

Uteri were dehydrated with increasing ethanol concentrations $(25,50,75$, and $100 \%, 20$ min each) and submersed in xylenes $(2 \times, 15 \mathrm{~min})$. The tissue was placed in melted paraffin wax overnight and embedded at room temperature, and the blocks were solidified at $-80^{\circ} \mathrm{C}$. Seven micrometer sections were cut with the Leica RM2145 microtome and dried overnight. Slides were then washed in xylenes, rehydrated with a decreasing ethanol gradient (100, 95, 85, 75, 50 and 20\%, 2 min each). Sections were then either used for immunofluorescence or stained with hematoxylin and eosin (H\&E). Briefly, sections were placed in Harris Modified Hematoxylin solution (Sigma) for $6 \mathrm{~min}$ and were then washed in running tap water for $20 \mathrm{~min}$. Sections were decolorized in 1\% acidic alcohol $(1 \mathrm{~s})$ and placed in a $1 \%$ sodium bicarbonate bluing agent $(3 \mathrm{~s})$ before being stained with Eosin (Sigma) for $15 \mathrm{~s}$. The slides were then dehydrated as previously described and mounted using permount.

\section{Embryo flushing and collection}

Females were mated with fertile CD1 males and the day of the vaginal plug was assigned as day 0.5. Embryos were collected from the uterus on day 3.5 in order to ensure that embryo transport to the uterus was not compromised.

\section{Immunofluorescence}

Whole uteri were dissected in PBS and fixed overnight at $4{ }^{\circ} \mathrm{C}$ in $4 \%$ paraformaldehyde (PFA)/PBS. Samples were dehydrated with increasing ethanol concentrations (25, 50, 75 and $100 \%, 20 \mathrm{~min}$ each) and submerged in xylenes (2x,15 min). The tissue was placed in melted paraffin wax (TissueTek) and xylenes $(1: 1)$ for $1 \mathrm{~h}$ at $60^{\circ} \mathrm{C}$, followed by $100 \%$ paraffin overnight under a vacuum. Samples were embedded at room temperature and blocks were solidified at $-20^{\circ} \mathrm{C}$. Sevenmicrometer sections were cut with a Leica RM2145 microtome and dried overnight. Slides were then washed in xylenes and rehydrated with a decreasing ethanol gradient $(100,95,85,75$, $50,20 \% ; 2$ min each). Samples were permeabilized with PBT $(0.2 \% \mathrm{BSA}, 2.5 \%$ TritonX-100 $\mathrm{PBS})$ for $10 \mathrm{~min}$ and incubated with primary antibody at $4{ }^{\circ} \mathrm{C}$ overnight: E-cadherin (Abcam ab15148, 1:150), Nodal (Santa Cruz Sc-28913, 1:100), Sox9 (Santa Cruz Sc-20095; 1:50), Foxa2 (Abcam ab108422 1:500) or GFP (Abcam ab13970, 1:500). Slides were washed with $0.1 \%$ PBS-Tween-20 three times before blocking with $5 \%$ heat-inactivated serum in PBT for $1 \mathrm{~h}$ at room temperature. Following several washes with $0.1 \%$ PBS-Tween 20, slides were incubated with a secondary goat anti-rabbit Alexa 488 (Life Technologies A11008 1:500) or goat anti-chicken Alexa 555 (Abcam ab150170 1:500) for $1 \mathrm{~h}$ at room temperature. Slides were then washed, counter stained with DAPI and mounted with Mowiol 4-88.

\section{Assessment of $E_{2}$ response}

Control and mosaic females were sedated, both uterine horns were ligated and females were ovariectomized. Females were 
given 2 weeks to recover, after which each female received three daily IP injection of $17 \beta$-estradiol ( $100 \mathrm{ng} / \mathrm{mouse} /$ day) for 3 days. Uteri from the females were then dissected for RNA extraction.

\section{Reverse transcription and real-time PCR}

RNA was collected using TRIzol extraction and RNeasy Mini Kit (Qiagen Cat. No. 74104). cDNA was then synthesized using the QuantiTect Reverse Transcription Kit (Qiagen Cat. No. 205311). Real-time PCR was performed using the RotorGene SYBR Green PCR Kit (Qiagen Cat. No. 204074) as per the manufacturer's protocol. The primers used are listed in (Supplementary Table 1, see section on supplementary data given at the end of this article).

\section{Detection of $\beta$-galactosidase activity}

Uteri were collected on gestational days 3.5 and 4.5 from control and mutant females. Uteri were washed in PBS and fixed in $4 \%$ PFA/PBS for $30 \mathrm{~min}$. Uteri were subsequently washed three times for $15 \mathrm{~min}$ each in wash buffer $(100 \mathrm{mM}$ sodium phosphate, $2 \mathrm{mM} \mathrm{MgCl} 2,0.02 \%$ Nonidet P-40, $0.01 \%$ sodium deoxycholate, $\mathrm{pH}$ 7.3). $\beta$-Galactosidase activity was detected by incubating the uteri in staining solution $\left(1 \mathrm{mg} / \mathrm{mL} \mathrm{X}\right.$-Gal, $\left.5 \mathrm{mM} \mathrm{K}_{3} \mathrm{Fe}(\mathrm{CN})_{6}, 5 \mathrm{mM} \mathrm{K}_{4} \mathrm{Fe}(\mathrm{CN})_{6}\right)$ at $37^{\circ} \mathrm{C}$ overnight. Uteri were then washed and fixed overnight in $4 \%$ formaldehyde/PBS at $4{ }^{\circ} \mathrm{C}$ before whole-mount photography and histological examination.

\section{Statistical analysis}

Figure data are presented as mean \pm S.E.M. The sample number for each figure is listed in the figure legend. Statistical analysis comparing experimental groups was performed using a twotailed Student $t$-test. Calculations were performed using the GraphPad software. $P$ values less than 0.05 were considered statistically significant.

\section{Results}

\section{Porcn mosaicism results in reduced fertility}

Porcn is located on the $\mathrm{X}$ chromosome in both mice and humans. Because one $X$ chromosomes is silenced due to random $X$ inactivation, heterozygous Porcn mutants are natural mosaic. We took advantage of the location of Porcn on the $\mathrm{X}$ chromosome to address its role in the female reproductive tract. We generated mosaic reproductive tract females by crossing Porch ${ }^{\text {flox/+ }}$ females with progesterone receptor $(\mathrm{Pgr})$-Cre males $\left(\mathrm{Pgr}^{\mathrm{Cre} /+}\right)$ to generate $\operatorname{PorCn}^{\Delta /+} ; \mathrm{Pgr}^{\mathrm{Cre} /+}$ females that will be referred to Porcn mosaic. A 6-month breeding trial was conducted on control females (Porch flox/flox $)$ and Porcn mosaic females to determine if mosaic females had any fertility issues. Five breeding pairs were set up for both Porch flox/flox and Porcn mosaic females. Interestingly, Porcn mosaic females were extremely subfertile. During the breeding trial, Porch flox/flox each had an average of four litters, whereas only one Porcn ${ }^{\text {mosaic }}$ female gave birth to a first litter of three pups and a second litter of one pup. The four other Porcn ${ }^{\text {mosaic }}$ females failed to become pregnant during this time period. Examination of vaginal smears from both Porcn $^{\text {flox/flox }}$ and Porcn mosaic females for 18 consecutive days indicated that both control and mosaic females cycled regularly (Supplementary Fig. 1). Furthermore, ovulation, fertilization, early embryo development and oviductal transport of embryos seemed to be normal, as normal and healthy embryos were present in the uterus on day 3.5 of gestation (Table 1). These results suggest that the infertility was due to a uterine defect.

\section{Porcn mosaicism results in variable endometrial gland size}

Upon examination of Porcn ${ }^{\text {mosaic }}$ uteri, a phenotype of variable severity was observed. While some Porcn ${ }^{\text {mosaic }}$ uteri looked similar to Porch flox/flox controls (Fig. 1A, B, C, D, E, F and G), others were larger, cystic and more transparent (Fig. 1G). Histological examination of the Porcn ${ }^{\text {mosaic }}$ uteri confirmed the wide spectrum of phenotype severity and revealed the presence of large fluid-filled cysts that were not present in Porcn flox/flox uteri (Fig. 1B, C, D, E and F). Measurement of uterine horn length and weight were comparable between 10-weekold Porcn flox/flox and Porcn mosaic uteri (Fig. 2A and B). Although gland number was comparable between the two genotypes (Fig. 2C), the average gland size was significantly greater in the Porcn mosaic uteri compared to Porcn $^{\text {flox/flox }}$ (Fig. 2D). It is important to mention that the gland size was correlated to phenotype severity; in the most severe cases, all glands were extremely cystic and enlarged, whereas milder phenotypes included both enlarged and regular-sized glands. These results were confirmed by quantifying uterine width. Average uterine width was not significantly different between Porch flox/ flox and pooled Porcn mosaic uteri. However, uterine width was significantly different between Porcn ${ }^{\text {mosaic }}$ uteri that exhibited a phenotype compared to Porch ${ }^{\text {flox/flox }}$ uteri (Fig. 2E).

In order to confirm whether enlarged cysts were derived from glandular epithelium, and if so whether they maintained any glandular identity, we performed a series of immunohistological stainings with several epithelial and glandular markers. Staining for the epithelial marker E-cadherin was visible in the luminal and glandular compartments in both Porch flox/flox and Porcn mosaic (Fig. $3 \mathrm{~A}$ and B), confirming an epithelial

Table 1 Porcn mosaic females have severe fertility issues. Data are presented as mean \pm S.E.M.

\begin{tabular}{lccc}
\hline Genotype & Liter size & Cycle length & $\begin{array}{c}\text { Embryos } \\
\text { collected }(E 3.5)\end{array}$ \\
\hline Porcn $^{\text {flox } / \text { flox }}$ & $13.2 \pm 1.01(n=5)$ & $5.23 \pm 0.61(n=13)$ & $10.3 \pm 0.71(n=6)$ \\
Porcn $^{\Delta /+}$ & $0.25 \pm 0.16(n=20)$ & $4.08 \pm 0.47(n=13)$ & $9.0 \pm 0.58(n=4)$ \\
& $P<0.0001$ & $P=0.096$ & $P=0.22$ \\
\hline
\end{tabular}

Reproduction (2018) 155 93-102 

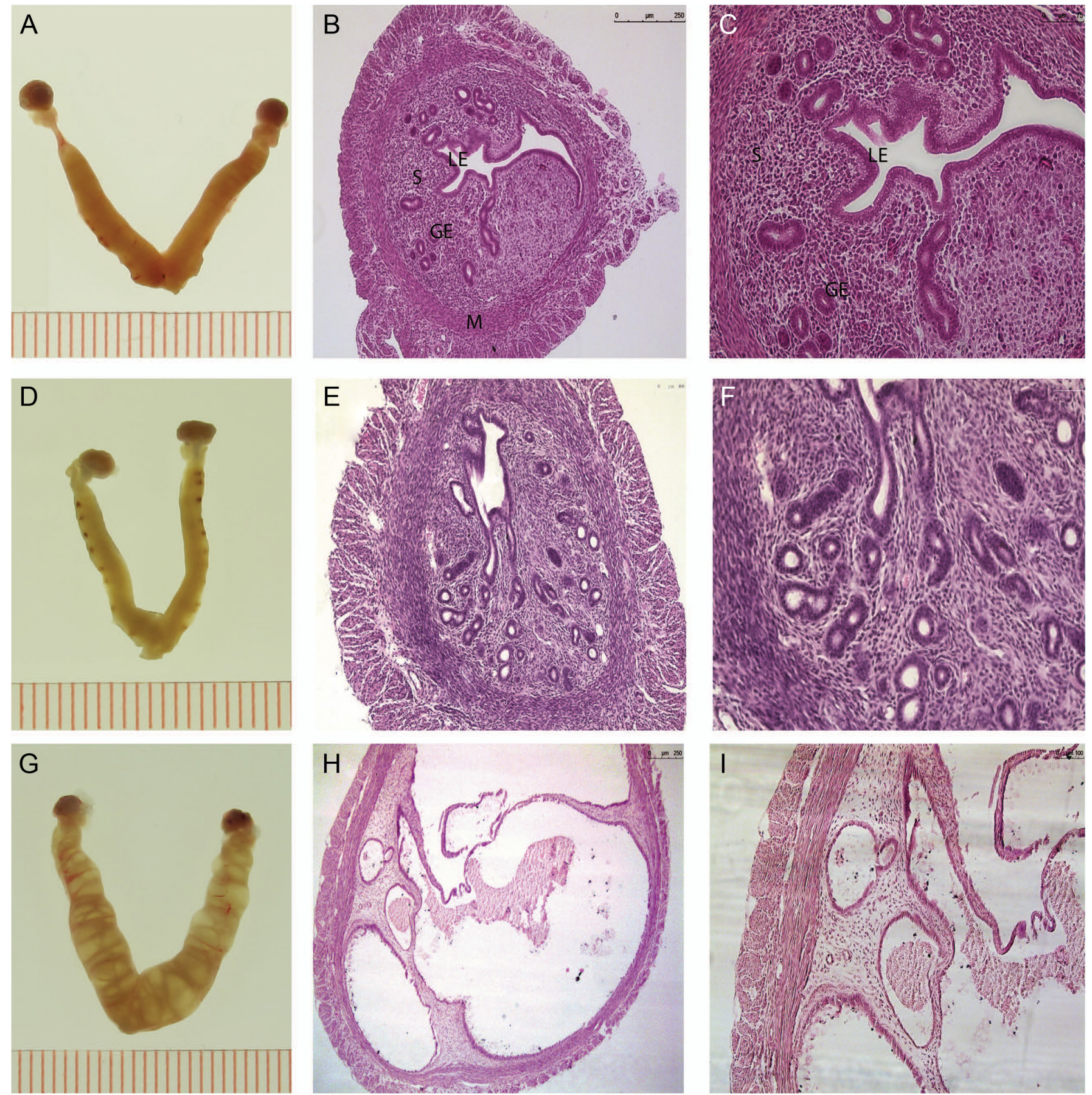

Figure 1 Porcn mosaic uteri exhibit a wide range of phenotypes. (A, B and C) Whole-mount and transverse sections Porcn floxed control uteri $(n=8)$. (D, E and F) Whole-mount and transverse sections of histologically normal Porcn mosaic uteri $(n=10)$. (G, H and I) Whole-mount and transverse sections of Porcn mosaic uteri showing an enlarged gland phenotype $(n=10)$. Uteri were analyzed at 10 weeks of age.

identity of the cysts. Staining for Nodal, a protein secreted by the endometrial glands (Fig. 3C), was also present in large cysts in the Porcn mosaic uteri (Fig. 3D). Staining for SOX9, a transcriptional factor expressed in the glandular epithelium, was localized to the nuclear compartment in the epithelium of control uteri (Fig. 3E) and was expressed in cells lining large cysts but was localized to the cytoplasm, and not the nucleus, in the Porcn mosaic (Fig. 3F). Interestingly, staining for the glandular epithelium-specific marker FOXA2 (Fig. 3G) showed that only a subset of cells lining the cysts where FOXA2-positive (Fig. $3 \mathrm{H}$ ). This suggests that the glandular identity of these enlarged structures within the endometrium is maintained by only a subset of cells lining the enlarged cysts, whereas all cells maintain E-cadherin expression. 

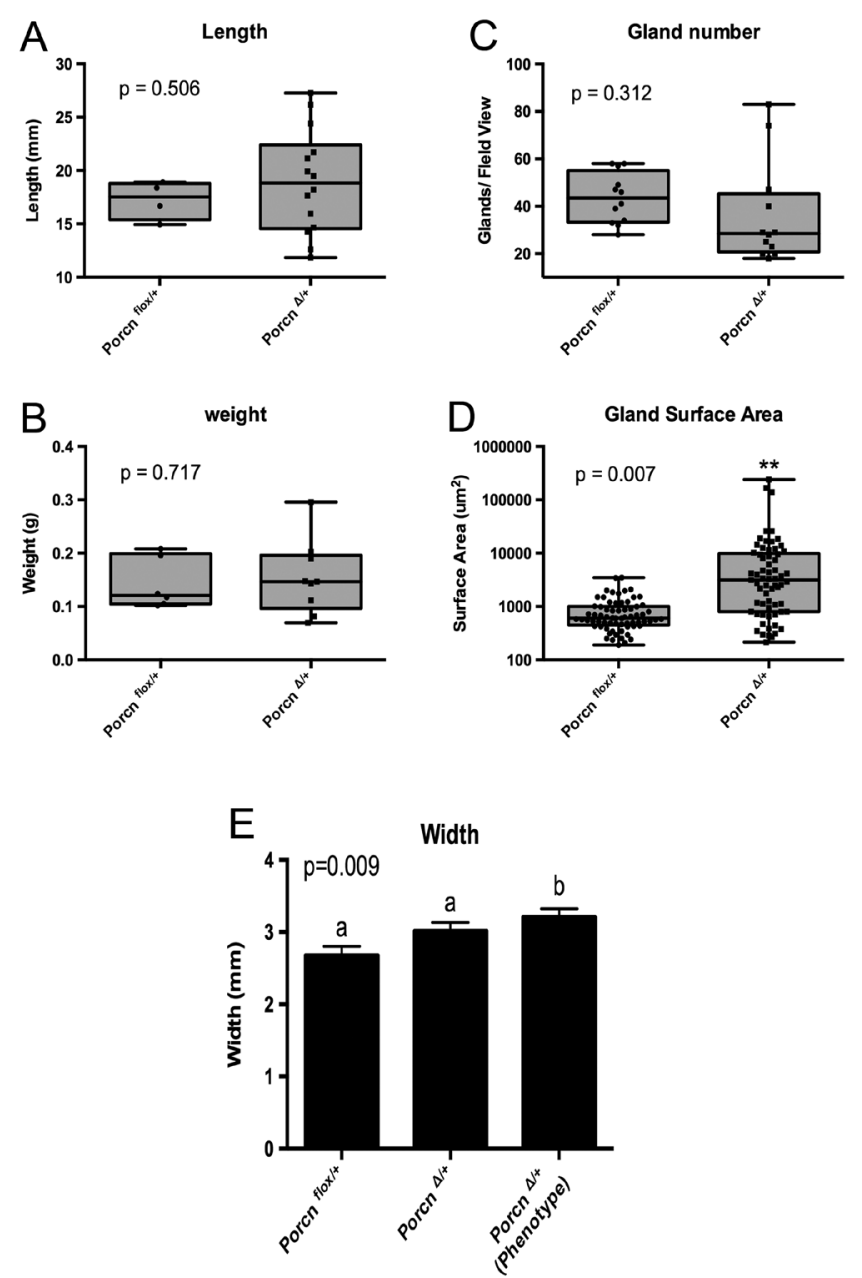

Figure 2 Quantification of Porcn mosaic uteri metrics reveals an increase in gland size. Quantification of uterine (A) length $(n=4,14)$, (B) weight $(n=6,9)$, (C) gland number $(n=12)$ and (D) gland surface area $(n=69,65)$ of Porcn control and mosaic uteri. (E) Quantification of uterine width of Porcn control, mosaic and phenotype-presenting mosaic uteri $(n=36,46,46)$. All uteri were examined at 10 weeks of age in these experiments.

\section{Enlarged glands are surrounded by a population of Porcn mutant stromal cells}

Given that only some cells within the endometrial glands maintained their glandular identity, we examined whether there was an association between phenotype severity and location of mutant cell populations in the uterus. In order to answer this question, we crossed a mouse strain carrying a transgenic GFP allele on the $X$ chromosome with our Porcn mouse strain. By doing so, we obtained mice that had LoxP sites flanking the Porcn allele on one $X$ chromosome and a GFP transgene on the other $X$ chromosome. Since one $X$ chromosomes is randomly inactivated, we were able to distinguish between Porcn mutant and wildtype cells by examining the expression of GFP. Wildtype cells have an inactivated $\mathrm{X}$ chromosome containing the Porcn deletion and an active $X$ chromosome expressing the GFP transgene. Conversely, Porcn mutant cells have an inactivated $\mathrm{X}$ chromosome containing the GFP transgene and are GFP negative (Fig. 4A).

When staining the Porcn ${ }^{\text {mosaic }}$ uteri with an anti-GFP antibody, we observed that the enlarged cyst phenotype corresponded with a large degree of mosaicism in both the luminal and stromal cell populations (Fig. 4B). No bias was observed in the genetic makeup of the epithelium of the cysts, as enlarged cysts were composed of a combination of both Porcn mutant and wildtype cells (Fig. 4C). Conversely, the stromal cells surrounding enlarged glands were mainly Porcn mutant cells, whereas normal-sized endometrial glands were surrounded by some Porcn wildtype cells (Fig. 4D). Our results suggest that Porcn wildtype stroma secreting stromal Porcupine-dependent Wnts is required to control endometrial gland size. In the absence of stromal Wnt secretion by Porcn mutant stromal cells, endometrial glands form cyst-like structures.

\section{Proliferation and apoptosis are altered in Porcn mosaic uteri}

Following the characterization of glandular identity, we turned our attention to determining how these glandderived cysts become enlarged. We hypothesized that an increase in luminal and glandular epithelial proliferation causes the formation of these large cystic structures. Staining of Porcn $n^{\text {flox/flox }}$ and Porcn ${ }^{\text {mosaic }}$ uteri at the diestrus stage with the proliferation marker PCNA showed that most of the endometrial glands in Porcn ${ }^{\text {flox }}$ flox females did not stain positive for PCNA (Fig. 5A). However, most endometrial glands within the PorCn ${ }^{\text {mosaic }}$ uteri, including the enlarged cysts, stained positive for PCNA (Fig. 5B). In addition to assessing the proliferative profile of the Porcn ${ }^{\text {mosaic }}$ uteri, we also examined the apoptotic profile during the estrus stage of the estrus cycle. Porcn flox/flox females exhibited higher levels of apoptosis of the LE compared to Porcn mosaic females (Fig. 5C and D). It is important to note that these proliferative and apoptotic profiles were visible in all females regardless of whether or not they presented with the phenotype. This indicates that endometrial glands within the Porcn ${ }^{\text {mosaic }}$ uteri may have a proliferative advantage compared to controls, and this may account for their increased size.

We next examined $\beta$-catenin/Tcf/Lef signaling in the uteri of Porcn ${ }^{\text {mosaic }}$ females at the diestrus stage by introducing our reporter transgene, TCF/Lef-LacZ, in the Porcn flox/flox and Porcn mosaic females. In Porcn flox/flox uteri, active $\beta$-catenin/Tcf/Lef-dependent signaling was observed in the LE and in the endometrial glands (Supplementary Fig. 2A). A similar pattern of active $\beta$-catenin/Tcf/Lef-dependent signaling was observed in Porcn ${ }^{\text {mosaic }}$ uteri (Supplementary Fig. 2B). Interestingly, although $\beta$-catenin/Tcf/Lef-dependent signaling was observed in smaller endometrial glands, no signaling 

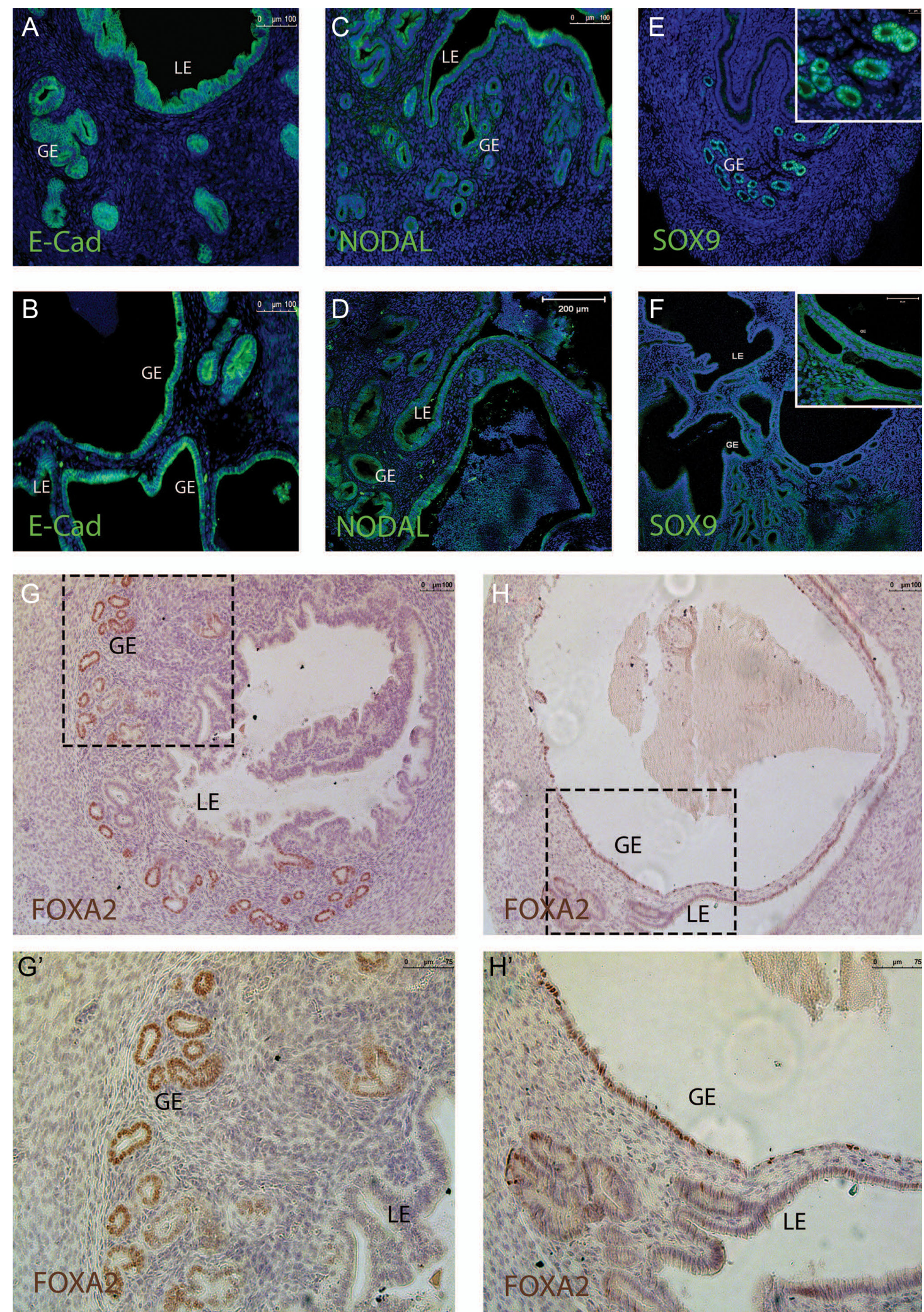

Figure 3 Uterine gland identity is compromised but not completely lost in the Porcn mosaic uterus. Immunofluorescence staining of epithelial and glandular marker (A and B) E-cadherin, (C and D) NODAL and (E and F) SOX9 on 10-week-old control and mosaic uteri, respectively. Immunohistochemical staining of the gland-specific marker $(\mathrm{G}$ and $\mathrm{H})$ FOXA2 on control and mosaic uteri, respectively. $\left(\mathrm{G}^{\prime}, \mathrm{H}^{\prime}\right)$ Higher magnification of panels $\mathrm{G}$ and $\mathrm{H}$, respectively ( $n=5$ each group). 
A
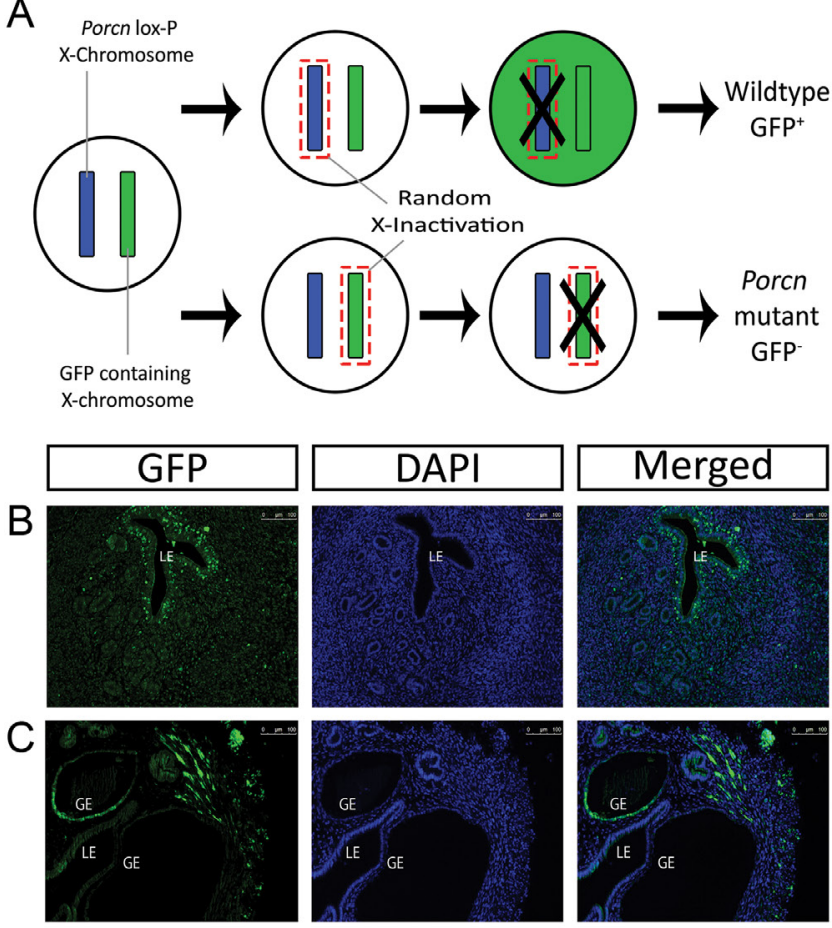

D

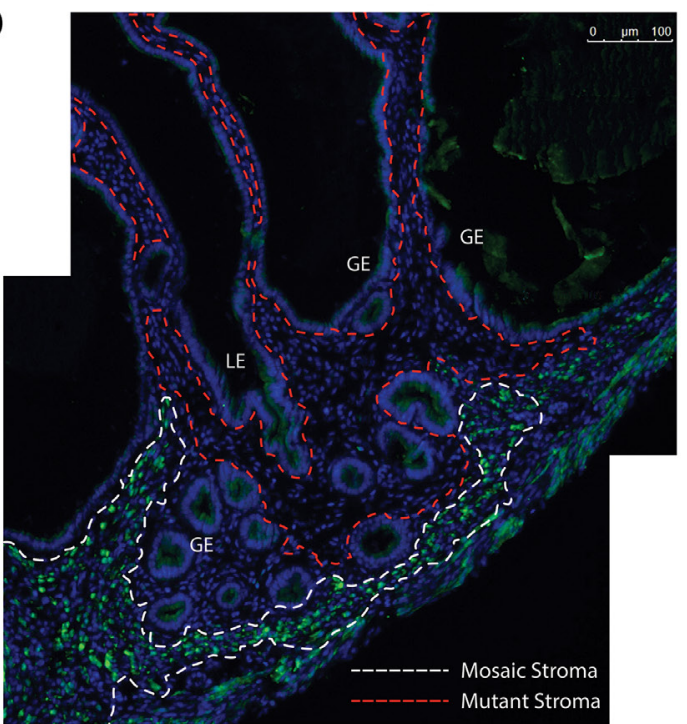

Figure $4 \mathrm{~A}$ skew in Porcn mosaicism within the uterine stroma is correlated to enlarged glands. (A) Schematic depiction of Porcn mosaicism through the process of random $\mathrm{X}$-inaction, leading to a $\mathrm{GFP}^{+}$wildtype cell or a GFP- mutant cell. Immunofluorescence staining of GFP on phenotype-presenting Porcn mosaic uteri showing a random pattern around the (B) luminal epithelium and (C) enlarged endometrial glands. In the Porcn mosaic uteri, the stroma surrounding the enlarged glands is mostly GFP negative, while normal-sized glands are surrounded by some GFP-positive stroma (D). All uteri examined were 15 weeks of age ( $n=6$ each group). LE, luminal epithelium; GE, glandular epithelium; S, stroma.

activity was detected in the epithelium of large cystic glands (Supplementary Fig. 2B). Thus, $\beta$-catenin/Tcf/ Lef-dependent signaling appeared to be unaffected
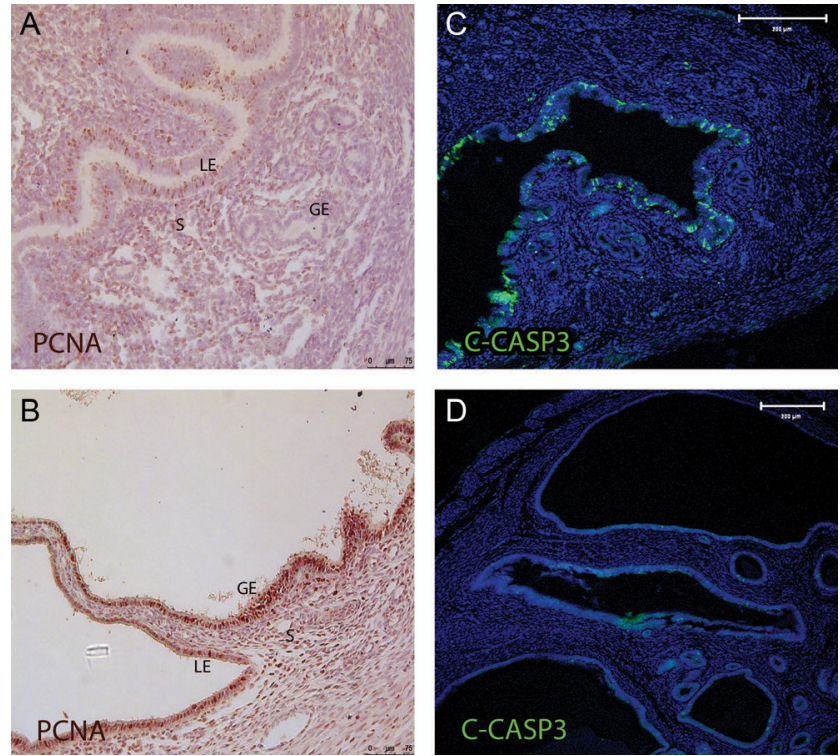

Figure 5 The luminal and glandular epithelium of the Porcn mosaic uteri are actively proliferating. (A and B) Immunohistochemical staining of the proliferation marker PCNA on control and mosaic uteri respectively during the diestrus stage. (C and $\mathrm{D})$ Immunofluorescence staining of the apoptosis marker CleavedCASPASE-3 on control and mosaic uteri respectively during the estrus stage. All uteri examined uteri were 10 weeks of age $(n=5$ each group).

in normal-sized glands but was absent in large cystic glands.

\section{Porcn mosaicism impairs $E_{2}$ response within the uterus}

Although Porcn mosaic females cycled regularly, produced viable embryos, and in most cases appeared histologically normal, these mice were infertile. Previous work has shown that the stabilization of one $\beta$-catenin allele $\left(C \operatorname{Cnn} b 1^{f(E \times 3) /+}\right)$ in the female reproductive tract results in an enlarged gland phenotype and a defect in estrogen response (Jeong et al. 2009). In order to address whether the estrogen response was altered in our mouse model, we ovariectomized control and histologically normal mosaic females supplemented with exogenous $\beta$-estradiol. We observed a significant decrease in the $\mathrm{E}_{2}$ response of mosaic females, as measured by uterine weight after a 3 -day period of $\beta$-estradiol injections, as compared to controls (Fig. 6A). We also measured relative levels of the estrogen-responsive genes Lactoferrin, Mucin-1 and Lif. We observed a 2-fold decrease in the expression levels of Mucin-1 and Lif but no difference in the expression of Lactoferrin (Fig. 6B, C and D).

\section{Porcn mosaicism results in aberrant Tcf/Lef signaling during the pre-implantation window}

We next examined $\beta$-catenin/Tcf/Lef signaling during the window of implantation, as we have previously 

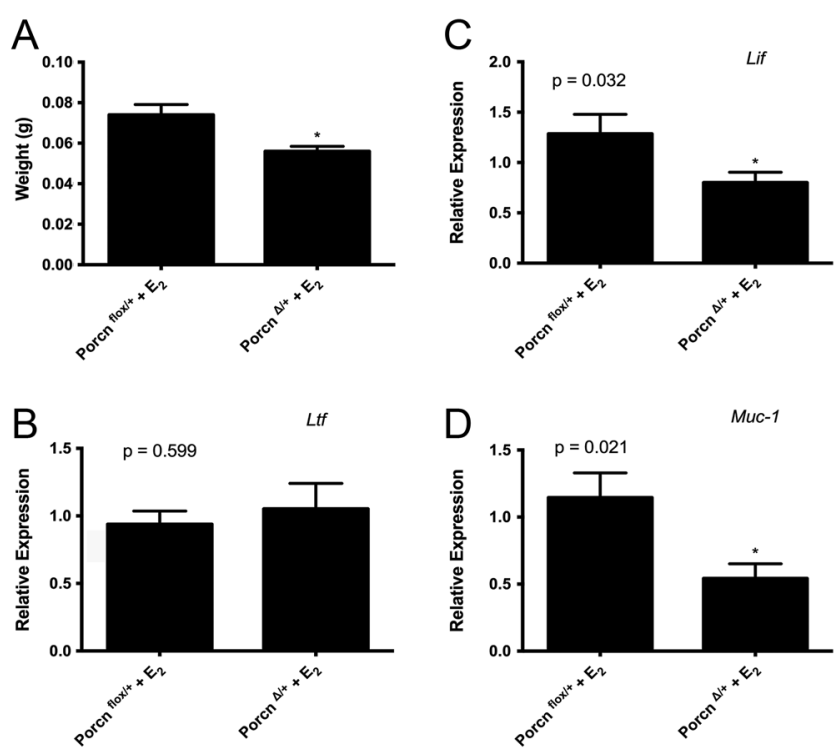

Figure $6 E_{2}$ response in Porcn mosaic females is slightly impaired. (A) Uterine weight quantification of Porcn control and mosaic females after ovariectomies and $E_{2}$ supplementation. (B, C and D) Comparative analysis of relative expression levels of $L t f$, $L i f$ and Muc-1 between control and mosaic uteri. All uteri examined were 10 weeks of age ( $n=5$ each group).

demonstrated its importance in embryo implantation (Mohamed et al. 2005). On gestational day 4, the presence of the embryo is known to activate Tcf/ Lef-dependent signaling in a banding pattern on the anti-mesometrial side of the LE. To examine Tcf/Lefdependent signaling, we again used Porch flox/flox and Porcn ${ }^{\text {mosaic }}$ females carrying the TCF/Lef-LacZ reporter construct. In Porcn flox/flox uteri, active Tcf/Lef-dependent signaling was observed in a banding pattern along the uterus and was specifically located in the LE of the antimesometrial side where an embryo was present, along with a small subset of endometrial glands (Fig. 7A, B and C). Interestingly, Tcf/Lef-dependent signaling in Porcn ${ }^{\text {mosaic }}$ uteri was present throughout the uterus rather than in a banded pattern (Fig. 7D). Signaling activity was no longer restricted to the anti-mesometrial side of the epithelium but was located in multiple random spots in the luminal and glandular epithelium, as well as underlying stroma among the mesometrial-antimesometrial (M-AM) axis (Fig. 7E and F). Furthermore, signaling was observed in certain regions of the stroma adjacent to the LE. These results suggest that Tcf/Lef signaling is deregulated, even in histologically normal Porcn $^{\text {mosaic }}$ uteri.

\section{Discussion}

Since Porcn is located on the $\mathrm{X}$ chromosome, heterozygous mutants present as natural mosaics due to random $X$-inactivation. We have shown most of these females exhibit hyperplasia of the uterine glands, whereas the uteri of some heterozygous females look morphologically normal. The large cystic uterine glands appear to proliferate excessively and have a significantly lower number of apoptotic luminal epithelial cells during the estrus stage of the estrus cycle. The increase in proliferation activity of the LE may cause hyperplasia of endometrial glands thus leading to the formation of large cystic glands.

The enlarged gland phenotype comes in contrast to the phenotype of the uterine Porcn mutant uteri where Cre recombinase is driven by the progesterone receptor promoter $\left(\operatorname{PorCn}^{\Delta / \Delta}\right.$ ) (Farah et al. 2017). Porcn ${ }^{\Delta / \Delta}$ are able to develop glands normally during the early stages of life, but these glands soon regress and are absent postpubertally. In addition, ablation of porcupine activity in the LE alone, by means of a Lactoferrin promoter-driven Cre recombinase $\left(\operatorname{PorCn}^{\mathrm{Epi} \Delta / \Delta}\right)$, results in the formation of endometrial glands in the adult uterus (Farah et al. 2017). The disparity between these three phenotypes may be explained by the necessity of precise regulation of distinct Wnts within the different compartments of the uterus. The absence of endometrial glands in $\operatorname{Porcn}^{\Delta / \Delta}$ mutants clearly demonstrates the requirement of Wnt signaling in adenogenesis (Farah et al. 2017). Furthermore, presence of endometrial glands in Porcn ${ }^{\text {Epi } \Delta / \Delta}$ mutants suggests that stromal Wnts are able to compensate for the absence of epithelial Wnts in endometrial gland formation (Farah et al. 2017). Finally, analysis of Porcn ${ }^{\text {mosaic }}$ demonstrates that stromal Wnts are required to regulate gland size and, in the absence of stromal Wnts, endometrial glands continue to proliferate and form epithelial-like cysts.

How stromal Wnts regulate endometrial gland proliferation is not clear. To date, large cystic endometrial glands have been observed when Sox9 is overexpressed and when one $\beta$-catenin allele is stabilized $\left(C \operatorname{ctnn} b 1^{f(E \times 3) /+}\right)$ in the female reproductive tract (Gonzalez et al. 2016). Overexpression of Sox9 in the female reproductive tract was sufficient to cause endometrial gland hyperplasia in an age-dependent manner (Gonzalez et al. 2016). Interestingly, active proliferation of most of the Sox-9-positive glands was not significantly different than controls except for small focal areas within the endometrial gland. It was postulated that these proliferating cells may be responsible for hyperplasia. In contrast to Sox 9 overexpression, we demonstrated that in Porcn mosaic, there was an increase in proliferation and a decrease in apoptosis throughout the glandular epithelium, which is likely responsible for the endometrial gland hyperplasia. Interestingly, Sox9 was expressed in the cytoplasm of glandular epithelial cells in Porcn mosaic but localized to the nucleus in Porcn flox/flox controls. What accounts for this difference in localization is unknown. The difference in proliferation and absence of nuclear Sox9 suggests that the hyperplasia observed in Porcn ${ }^{\text {mosaic }}$ females is not due to Sox9 activity. 

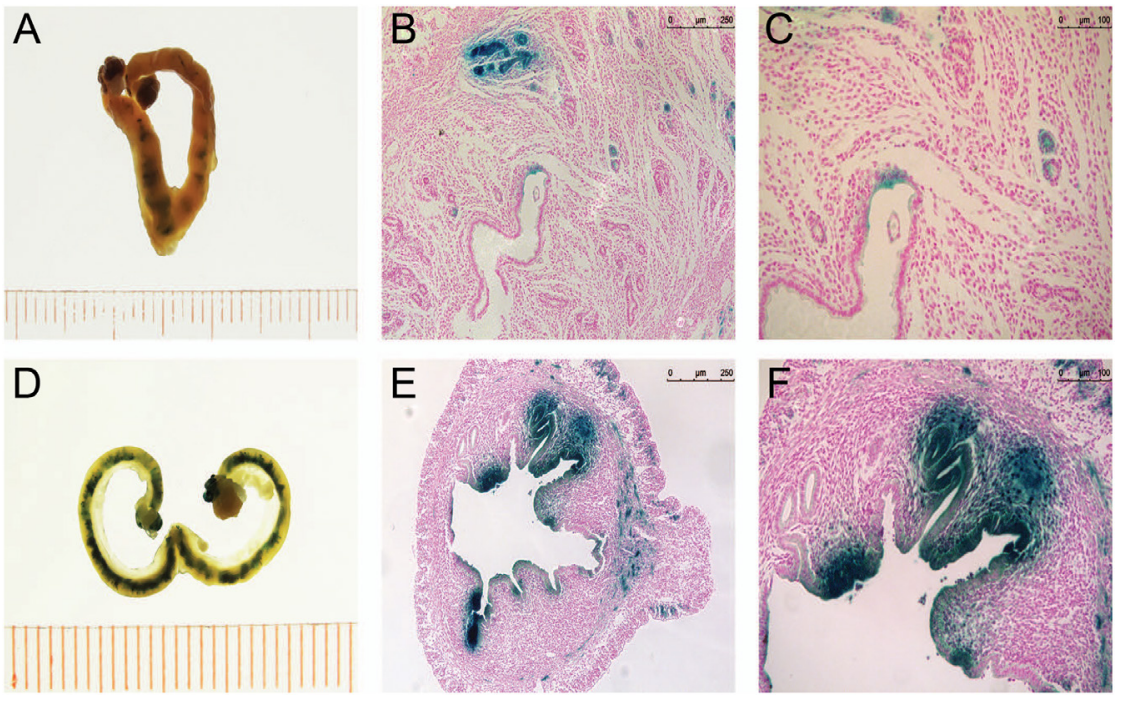

Figure 7 Porcn mosaicism leads to aberrant Tcf-Lef signaling during the window of implantation. (A, B and C) Whole-mount and sagittal sections of Tcf-lacZ stained Porcn floxed control uteri on gestational day 4. (D, E and F) Whole-mount and transverse sections of Tcf-lacZ stained, histologically normal Porcn mosaic uteri on gestational day 4. All uteri examined were 10 weeks of age $(n=5)$.
Stabilization of one $\beta$-catenin allele $\left(C \operatorname{Cnn} b 1^{f([\times 3) /+}\right)$ in the female reproductive tract was also previously shown to lead to gland hyperplasia as well as an increase in the levels of cyclin (Jeong et al. 2009). We have demonstrated that active $\beta$-catenin/Tcf/Lef-dependent signaling in the non-pregnant uterus of Porcn ${ }^{\text {mosaic }}$ females is similar in normal-sized glands to that of Porcn flox/flox controls, but is absent in the large cystic glands (Supplementary Fig. 2). This suggests that either $\beta$-catenin signaling is not involved in gland hyperplasia and was affected in Porcn ${ }^{\text {mosaic }}$ or that $\beta$-catenin signaling is involved but does not signal through Tcf/Lef and was not detected by our reporter transgene. We currently cannot distinguish between these two possibilities but favor the hypothesis that stromal $\beta$-catenin signaling is not involved in gland hyperplasia in Porcn ${ }^{\text {mosaic }}$.

Interestingly, Porc ${ }^{\text {mosaic }}$ females with morphologically normal uteri had severely reduced fertility compared to Porch flox/flox controls. This suggests that subfertility within the normal uteri was due to changes within the porcupine-dependent Wnt signaling pathway as a direct result of the mosaic nature of the porcupine deletion. $\beta$-catenin/Tcf/Lef signaling on day 3.5 was no longer restricted to the LE adjacent to the implanting embryo but was seen in patches throughout the luminal epithelium. Since precise $\beta$-catenin signaling is essential for implantation (Mohamed et al. 2005), aberrant signaling within the morphologically normal heterozygous females is likely one of the causes for the subfertility phenotype. This increase in $\beta$-catenin/Tcf/Lef signaling is likely due to a defect in the stroma as signaling in the lumen of Porcn ${ }^{\mathrm{Ep} i} \Delta / \Delta$, which lack epithelial porcupine activity, is mislocalized but is not significantly increased (Farah et al. 2017). Thus, stromal Wnts may also be responsible for restricting $\beta$-catenin/Tcf/Lef signaling in the epithelium. Groups of stromal cells lacking Porcn are sufficient to deregulate $\beta$-catenin/Tcf/Lef signaling in the epithelium.
It has previously been demonstrated that either the deletion of $\beta$-catenin or the stabilization of one $\beta$-catenin allele leads to defects in response to estrogen in the uterus of mice (Jeong et al. 2009). We have also demonstrated that the response to estrogen is also altered in the uterus of Porcn mosaic females. Our results confirm the role of the Wnt signaling pathway in modulating the response to estrogen. However, the mechanism by which Wnts interact and influence estrogen signaling still remains elusive. It is possible that Wnts modulate the response to estrogen by either acting directly downstream of the estrogen signaling pathway or by indirectly modulating the ability of cells to respond to estrogen. Further work is required to distinguish between these two possibilities. The altered response to estrogen may also contribute to the decreased fertility in Por $n^{\text {mosaic }}$ females. Deregulated Tcf/Lef signaling in the LE and the impaired estrogen response may lead to defects in proper embryo implantation and infertility in Porcn ${ }^{\text {mosaic }}$ females.

Our study has shed light on the importance of precise spatial expression and activity of porcupine-dependent Wnt signaling within the uterus. We demonstrated a crucial role for stromal $\mathrm{Wnt}$ signaling in regulating endometrial gland proliferation. Furthermore, we demonstrated that porcupine-dependent Wnt signaling is required for proper estrogen response and the regulation and localization of Tcf/Lef signaling in the uterus. Finally, given that focal dermal hyperplasia $(\mathrm{FDH})$ is caused by porcupine heterozygosity in humans (Barrott et al. 2011), our model may provide some useful insights for exploring the genetic basis of infertility in FDH patients.

\section{Supplementary data}

This is linked to the online version of the paper at https://doi.org/10.1530/REP-17-0436. 


\section{Declaration of interest}

The authors declare that there is no conflict of interest that could be perceived as prejudicing the impartiality of the research reported.

\section{Funding}

This research was funded by a grant from the Canadian Institutes of Health Research.

\section{Acknowledgments}

The authors would like to thank Dr Lisa Starr for her input and aid in the editing of this manuscript.

\section{References}

Barrott JJ, Cash GM, Smith AP, Barrow JR \& Murtaugh LC 2011 Deletion of mouse Porcn blocks Wnt ligand secretion and reveals an ectodermal etiology of human focal dermal hypoplasia/Goltz syndrome. PNAS 108 12752-12757. (https://doi.org/10.1073/pnas.1006437108)

Bartol FF, Wiley AA, Floyd JG, Ott TL, Bazer FW, Gray CA \& Spencer TE 1999 Uterine differentiation as a foundation for subsequent fertility. Journal of Reproduction and Fertility: Supplement 54 287-302.

Biechele S, Cockburn K, Lanner F, Cox BJ \& Rossant J 2013 Porcndependent Wnt signaling is not required prior to mouse gastrulation. Development 140 2961-2971. (https://doi.org/10.1242/dev.094458)

Cha J \& Dey SK 2014 Cadence of procreation: orchestrating embryouterine interactions. Seminars in Cell and Developmental Biology 34 56-64. (https://doi.org/10.1016/j.semcdb.2014.05.005)

Cha JM \& Dey SK 2015 Reflections on rodent implantation. Advances in Anatomy, Embryology and Cell Biology 216 69-85.

Cooke PS, Spencer TE, Bartol FF \& Hayashi K 2013 Uterine glands: development, function and experimental model systems. Molecular Human Reproduction 19 547-558. (https://doi.org/10.1093/molehr/ gat031)

Dunlap KA, Filant J, Hayashi K, Rucker EB 3rd, Song G, Deng JM, Behringer RR, DeMayo FJ, Lydon J, Jeong JW et al. 2011 Postnatal deletion of Wnt7a inhibits uterine gland morphogenesis and compromises adult fertility in mice. Biology of Reproduction $\mathbf{8 5}$ 386-396. (https://doi.org/10.1095/biolreprod.111.091769)

Farah O, Biechele S, Rossant J \& Dufort D 2017 Porcupine-dependent Wnt signaling controls stromal proliferation and endometrial gland maintenance through the action of distinct WNTs. Developmental Biology 422 58-69. (https://doi.org/10.1016/j.ydbio.2016.11.023)

Fazleabas AT, Kim JJ \& Strakova Z 2004 Implantation: embryonic signals and the modulation of the uterine environment--a review. Placenta 25 (Supplement A) S26-S31. (https://doi.org/10.1016/j. placenta.2004.01.014)

Filant J \& Spencer TE 2014 Uterine glands: biological roles in conceptus implantation, uterine receptivity and decidualization. International
Journal of Developmental Biology 58 107-116. (https://doi.org/10.1387/ ijdb.130344ts)

Franco HL, Dai D, Lee KY, Rubel CA, Roop D, Boerboom D, Jeong JW, Lydon JP, Bagchi IC, Bagchi MK, et al. 2011 WNT4 is a key regulator of normal postnatal uterine development and progesterone signaling during embryo implantation and decidualization in the mouse. FASEB Journal 25 1176-1187. (https://doi.org/10.1096/fj.10-175349)

Gonzalez G, Mehra S, Wang Y, Akiyama H \& Behringer RR 2016 Sox9 overexpression in uterine epithelia induces endometrial gland hyperplasia. Differentiation 92 204-215. (https://doi.org/10.1016/j. diff.2016.05.006)

Gray CA, Bartol FF, Tarleton BJ, Wiley AA, Johnson GA, Bazer FW \& Spencer TE 2001 Developmental biology of uterine glands. Biology of Reproduction 65 1311-1323. (https://doi.org/10.1095/ biolreprod65.5.1311)

Hadjantonakis AK, Gertsenstein M, Ikawa M, Okabe M \& Nagy A 1998 Non-invasive sexing of preimplantation stage mammalian embryos. Nature Genetics 19 220-222. (https://doi.org/10.1038/893)

Jeong JW, Lee HS, Franco HL, Broaddus RR, Taketo MM, Tsai SY, Lydon JP \& DeMayo FJ 2009 beta-catenin mediates glandular formation and dysregulation of beta-catenin induces hyperplasia formation in the murine uterus. Oncogene 28 31-40. (https://doi.org/10.1038/ onc.2008.363)

Kadowaki T, Wilder E, Klingensmith J, Zachary K \& Perrimon N 1996 The segment polarity gene porcupine encodes a putative multitransmembrane protein involved in Wingless processing. Genes and Development 10 3116-3128. (https://doi.org/10.1101/gad.10.24.3116)

Mohamed OA, Jonnaert M, Labelle-Dumais C, Kuroda K, Clarke HJ \& Dufort D 2005 Uterine Wnt/beta-catenin signaling is required for implantation. PNAS 102 8579-8584. (https://doi.org/10.1073/ pnas.0500612102)

Payer B 2016 Developmental regulation of X-chromosome inactivation. Seminars in Cell and Developmental Biology 56 88-99. (https://doi. org/10.1016/j.semcdb.2016.04.014)

Sonderegger S, Pollheimer J \& Knofler M 2010 Wntsignalling in implantation, decidualisation and placental differentiation--review. Placenta 31 839-847. (https://doi.org/10.1016/j.placenta.2010.07.011)Soyal S M, Mukherjee A, Lee K Y-S, Li J, Li H, DeMayo F J Lydon J P 2005 Cremediated recombination in cell lineages that express the progesterone receptor. Genesis 41 58-66. (https://doi.org/10.1002/gene.20098)

van den Heuvel M, Harryman-Samos C, Klingensmith J, Perrimon N \& Nusse R 1993 Mutations in the segment polarity genes wingless and porcupine impair secretion of the wingless protein. EMBO Journal 12 5293-5302.

Wang H \& Dey SK 2006 Roadmap to embryo implantation: clues from mouse models. Nature Reviews Genetics 7 185-199. (https://doi. org/10.1038/nrg1808)

Received 21 July 2017

First decision 25 August 2017

Revised manuscript received 19 September 2017

Accepted 23 October 2017 